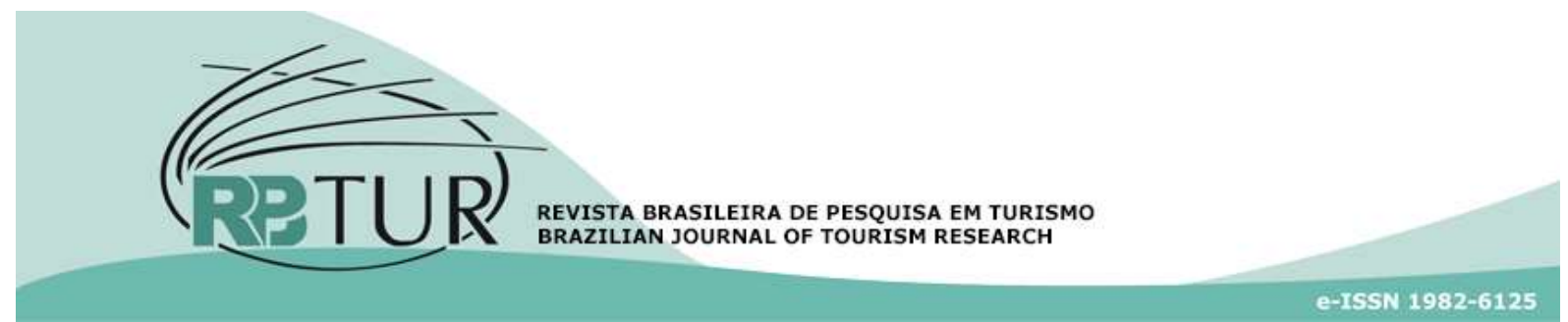

Artigo

DOI: http://dx.doi.org/10.7784/rbtur.v11i1.1153

\title{
Consumo de vinhos na cidade do Recife (Brasil): uma pesquisa da relevância dos atributos da bebida no momento da compra
}

\author{
Wine consumption in the city of Recife (Brazil): a study of the \\ relevance of the drink's attributes on the moment of purchase
}

Consumo de vinos en la ciudad de Recife (Brasil): un estudio sobre la relevancia de los atributos de la bebida al momento de

la compra

\author{
Nayara Diniz ${ }^{1}$ \\ Simone Almeida ${ }^{2}$ \\ Viviane Salazar ${ }^{3}$ \\ Anderson Gomes de Souza ${ }^{4}$
}

Resumo: Este estudo, de natureza teórico-empírica, diz respeito à identificação e análise de atributos relevantes no processo de decisão e compra de vinhos para consumidores do Recife. O modelo teórico adotado foi desenvolvido com base na identificação dos atributos do vinho avaliados mais frequentemente em estudos do comportamento do consumidor em diversos países. Consequentemente, com base no trabalho de Goodman et al. (2007), o grau de relevância de cada atributo foi classificado por meio da escala Best-Worst Scaling (BWS). Destarte, a abordagem metodológica adotada foi a quantitativa, cujo método de coleta de dados foi o questionário, adaptado de Forbes (2008) e Groot (2011), administrado em meio a um amostra de 100 consumidores. Os resultados mostraram que, preço, marca, prêmios, teor alcóolico e rótulo frontal atraente foram considerados atributos importantes para os consumidores. No entanto, os atributos decisivos para a decisão e compra eram outros: variedade da uva, país de origem do vinho, harmonização com alimentos e as informações no rótulo traseiro.

1 Universidade Federal de Pernambuco (UFPE), Recife, PE, Brasil. Coleta dos dados; análise e interpretação dos dados; redação do trabalho.

2 Universidade Federal de Pernambuco (UFPE), Recife, PE, Brasil. Concepção e desenho do trabalho científico; redação do trabalho.

3 Universidade Federal de Pernambuco (UFPE), Recife, PE, Brasil. Preparação do artigo científico, revisão crítica do trabalho.

4 Universidade Federal de Pernambuco (UFPE), Recife, PE, Brasil. Revisão crítica do trabalho; aprovação final.

Artigo recebido em: 23/03/2016. Artigo aprovado em: 13/10/2016. 
Palavras-chave: Comportamento do consumidor. Vinho. Atributos do produto. Escala Best-Worst.

\begin{abstract}
This study is a theoretical-empirical research on the identification and analysis of wine attributes' relevance in the purchase decision process to consumers from Recife. The theoretical framework was developed based on the identification of the most-frequently evaluated wine attributes in consumer behaviour studies in several countries. Subsequently, based on the work of Goodman et al. (2007), the relevance degree of each attribute was classified through the Best-Worst Scaling (BWS). The methodological approach was quantitative in nature, the data collection method was a questionnaire adapted from Forbes (2008) and Groot (2011) and completed by 100 consumers. The results show that price, brand, awards, alcohol by volume and attractive front label were considered important attributes by the consumers. However, the decisive attributes for the purchase decision were others: grape variety, country of origin of the wine, food harmonization and the information on the back label.
\end{abstract}

Keywords: Consumer behavior. Wine. Product attributes. Scale Best-Worst.

Resumen: El presente estudio es una investigación teórico-empírica sobre la identificación y análisis de la relevancia de los atributos del vino en la decisión de compra del producto de los consumidores de Recife. El marco teórico fue elaborado a partir de la identificación de los atributos del vino más evaluados en estudios sobre el comportamiento del consumidor en diversos países. Luego, en base al trabajo de Goodman et al. (2007) el grado de relevancia de cada atributo fue clasificado por medio del Best-Worst Scaling (BWS). El abordaje metodológico fue cuantitativo y el método de recolección de datos fue un cuestionario adaptado de Forbes (2008) y Groot (2011) y respondido por 100 consumidores. Los resultados indican que precio, marca, premiación, proporción alcohólica y etiqueta frontal atractiva fueron considerados atributos importantes por los consumidores. Sin embargo, los atributos determinantes para la decisión de compra del consumidor fueron otros: variedad de la uva, país de origen del vino, maridaje con la comida y la información contenida en la etiqueta posterior.

Palabras-clave: Comportamiento del consumidor. Vino; Atributos del producto. Escala Best-Worst.

\section{INTRODUÇÃO}

A análise do panorama mundial dos países consumidores de vinho indica que os Estados Unidos da América foi o país que mais consumiu vinho em 2013. Contudo, a Itália ficou em primeiro lugar no ranking dos maiores produtores de vinho superando outros países tradicionais na a fabricação da bebida, como Espanha e França. No mesmo ano, o Brasil ocupou o décimo sexto e o décimo nono lugar como consumidor e produtor mundial de vinho respectivamente (Wine Institute, 2013a, 2013b).

Em 2011, o cenário da vitivinicultura nacional finalizou o ano positivamente mesmo em meio à crise econômica da Europa que afetou o consumo dos brasileiros e ao câmbio desfavorável às exportações. Na ocasião, o consumo de vinhos finos tintos no país aumentou 7,5\% em relação a 2010 , conquistando o maior volume de vendas da década. Em termos de importações, houve um aumento de 2,3 milhões de litros em relação ao ano anterior. Já as exportações das vinícolas brasileiras associadas ao Wines of Brasil (projeto realizado pelo Instituto Brasileiro do Vinho e a Agência Brasileira de Promoção de Exportações e Investimentos), atingiram US\$ 3,06 milhões em 2011, 33,6\% a mais do que 0 ano anterior (Wines of Brasil, 2011; Wines of Brasil, 2012; IBRAVIN, 2012a; IBRAVIN, 2012b).

Mas por que os brasileiros estão con- 
sumindo mais vinho? Um dos motivos é o fenômeno da globalização que facilitou a entrada e saída de produtos no país, expandiu o comércio mundial e consequentemente ampliou a concorrência, provocando assim o melhoramento da qualidade dos produtos fabricados (Fernandes Neto, 2006).

Percebe-se que mudanças estão acontecendo no comportamento dos consumidores dessa bebida. Compreender esse comportamento é fundamental para o desenvolvimento desse mercado, afinal é o consumidor quem determina quais e quantos produtos serão adquiridos. Porém, identificar o perfil do consumidor e entender seu comportamento não é uma tarefa simples, visto que as pessoas apresentam diferentes desejos e necessidades, que são influenciados por diversos fatores (Kotler, 1998).

Dentre estes fatores, os atributos do produto constituem-se como elemento relevante para compreender a tomada de decisão do consumidor (Urdan \& Nagão, 2004, p. $3)$. Por isso, os pesquisadores de marketing tendem a explicar as preferências dos consumidores por meio da mensuração e análise da importância dos atributos do produto no momento da compra (Espartel, 1999).

Quando o consumidor compra um produto, ele analisa características como marca, preço, embalagem, entre outros, para tomar sua decisão final. Porém, o nível de influência que os atributos têm sobre o consumidor varia de acordo com o produto e o perfil das pessoas (Engel et al. 2000). No caso do vinho, a variedade de uva, o país de origem, o teor alcoólico e o preço são alguns dos atributos do produto que podem ser levados em consideração no momento da compra
(Forbes, 2008; Goodman et al., 2007; Lockshin et al., 2009). Pesquisa realizada por Forbes (2008) indica que o desconto no preço foi o atributo mais relevante para as mulheres no momento da compra de vinhos, enquanto que para os homens a região de origem da bebida foi o atributo mais mencionado.

Há ainda outros estudos que investigaram a relevância dos atributos do vinho no momento de sua compra (cf. Espartel, 1999; Groot, 2011), porém poucas são as pesquisas empíricas que abordam o comportamento do consumidor de vinho no Brasil. Diante dessa problemática, o objetivo geral desse estudo consiste em analisar a relevância dos atributos do vinho no processo decisório de compra desse produto para os consumidores do Recife, promovendo um maior entendimento no comportamento do consumidor de vinho no mercado nacional.

\section{MUDANÇAS NO CENÁRIO MERCA- DOLÓGICO DO VINHO}

Na década de 1990, a estabilização da economia brasileira e a abertura às importações, impulsionaram o consumo de vinhos finos entre a classe média (Malheiros, 2006). O aumento do poder de compra da classe média, aliado a uma maior oferta de produtos mundiais possibilitaram o acesso desse segmento social da população a produtos antes consumidos exclusivamente por consumidores de alta renda.

Entretanto, o processo de globalização não favoreceu o consumo de vinhos finos nacionais. Segundo Mello (2002) essa realidade se justificada devido à falta de hábito do 
brasileiro em beber vinho e a preferência por produtos importados. Na opinião do autor, para que os produtores nacionais reconquistem e aumentem sua participação no mercado brasileiro de vinhos finos é preciso direcionar esforços para incentivar o consumo e a mudança de hábito do consumidor, por meio de campanhas publicitárias, feiras e eventos (Mello, 2002).

Por outro lado, o processo de padronização do vinho - estratégia combatida pelos produtores e consumidores do chamado VeIho Mundo - tem contribuído para a democratização do consumo dessa bebida (Roese, 2008). A tecnologia industrial de produção empregada pela maioria dos vinícolas do Novo Mundo tem sido responsável pela padronização do produto e sua massificação no mercado. Deste modo, o mercado de vinho torna-se mais diversificado, pois ao mesmo tempo engloba o conceito de terroir dos vinhos do Velho Mundo e a padronização dos vinhos do Novo Mundo - países recém-produtores de vinho - assim como os vinhos orgânicos (Sloan, 2005) oferecendo ao consumidor uma maior variedade de produtos.

Além da variedade de produtos disponíveis no mercado, as normas de etiqueta para o consumo de vinhos finos - tais como o conhecimento sobre a temperatura adequada, o uso de taças específicas e noções que garantam a correta harmonização da bebida com a comida (Roese, 2008) - exigem do consumidor maior qualificação para sua "correta" compra e degustação. Sendo assim, o nível de exigência e de conhecimento desses consumidores tende a ser mais elevado (Mello, 2002).

Ademais, o vinho tornou-se assunto da moda. Há uma série de serviços e produtos disponíveis à classe média com objetivo de fornecer informações sobre a "arte do vinho". Cursos, aulas de degustação, cursos de formação de sommelier, programas na televisão e revistas especializadas estão disponíveis a esse novo consumidor de modo que ele também possa se tornar conhecedor do assunto (Zortea, 2010).

Diante dessas transformações no cenário da produção e do consumo de vinho, o comportamento do consumidor também vem sofrendo mudanças. Para entender e identificar as variações no comportamento das pessoas ao adquirirem vinhos e as futuras tendências de compra dessa bebida, as empresas precisam fazer um estudo sobre o comportamento do consumidor, assunto que será abordado na seção seguinte.

\section{O PROCESSO DE DECISÃO DO COM- SUMIDOR E OS ATRIBUTOS DOS PRODUTOS}

O processo de decisão do consumidor é dividido em estágios. Para alguns pesquisadores esse processo ocorre em cinco estágios (c.f Churchil \& Peter 2005; Las Casas, 2010; Hawkins et al. 2007; Shiffman \& Kanuk, 2009; Sheth et al., 2001): 1) Reconhecimento da necessidade ou problema; 2) Busca de informações ou busca pré-compra; 3) Avaliação de alternativas; 4) Decisão de compra ou compra; 5) Avaliação ou experiência pós-compra ou pós-consumo (Figura 1). Outros autores acrescentam mais duas etapas ao processo (c.f Blackwell et al. 2009), que são o consumo e o descarte. 


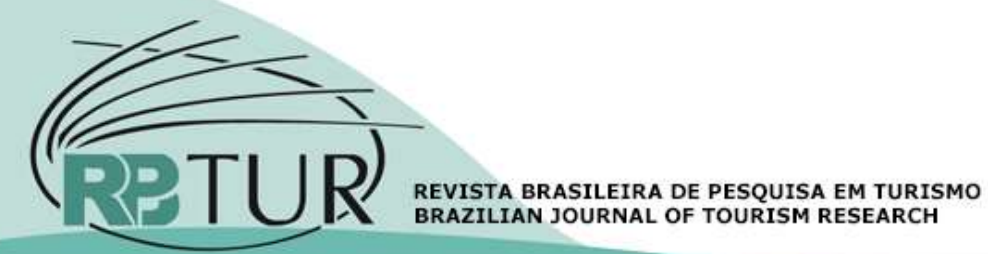

Figura 1 - Processo decisório do cliente

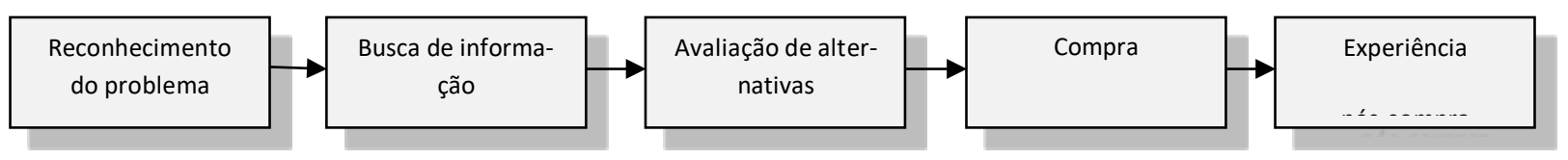

Fonte: Sheth (2001, p. 486)

Dentre estas etapas, duas delas parecem ser mais importantes para que possamos identificar e analisar os atributos que possuem maior relevância no processo decisório de compra de um vinho: a avaliação das alternativas e a de decisão de compra.

A primeira etapa do processo de decisão de compra é o reconhecimento do problema. $\mathrm{O}$ indivíduo reconhece o problema quando há uma necessidade a ser satisfeita, pois compara seu estado atual com o estado ideal e percebe que existe uma condição de carência, desconforto ou privação seja ela física ou psicológica (Stefano et al. 2008). Por exemplo, o consumidor percebe a necessidade de comer e beber algo quando sente sede ou fome, de comprar roupas novas quando se ver com poucas, de ligar para uma amiga quando sente saudades dela, ou de comprar um presente quando descobre que precisará comparecer a uma festa de aniversário no final de semana (Sheth et al., 2001).

Após reconhecer o problema, o consumidor procura informações sobre o produto - como seus atributos, funções, durabilidade e outros aspectos, em propagandas, opiniões de amigos, vendedores, etc. - para fundamentar sua tomada de decisão quanto aos produtos disponíveis (Kotler, 1998) e analisar as alternativas que indiquem o meIhor negócio (Las Casas, 2010). Como cada produto possui características diferentes, são os consumidores que determinam quais delas serão mais importantes na sua avaliação (Kotler, 1998). O consumidor pré-seleciona um pequeno número de marcas que considera aceitável e utiliza critérios para avaliar cada marca e tomar sua decisão de compra (Shiffman \& Kanuk, 2009). Em seguida, o cliente escolhe o produto que for avaliado positivamente e efetiva a compra (Sheth, 2001: 509). Por fim, o consumidor avalia sua satisfação geral com o produto, analisando sua experiência pós-compra, que serve como mecanismo de feedback para compras futuras (Kotler, 1998).

Os atributos do produto são considerados durante a avaliação de alternativas terceiro estágio do processo decisório do cliente. Esses elementos são os principais influenciadores na tomada de decisão de compra dos consumidores, por isso os pesquisadores de marketing têm a tendência de explicar as preferências dos consumidores pela análise dos atributos do produto. Ademais, por meio da mensuração do grau de relevância dos atributos para os clientes, o administrador de marketing, pode entender as crenças do consumidor a respeito de um determinado produto ou marca (Espartel, 1999).

A escolha baseada em atributos compreende a comparação de cada atributo do 
produto entre diversas marcas. Por isso, requer que os consumidores tenham conhecimento sobre os atributos específicos de cada produto no momento da compra, gerando uma decisão mais próxima da ótima (Hawkins et al., 2007).

Alguns atributos podem influenciar mais que outros (Engel et al. 2000) e esta influência varia de acordo com o tipo de produto e/ou do consumidor. A importância que cada atributo tem para o cliente refere-se às prioridades que este cliente dá a cada vantagem oferecida, visando a sua satisfação (Espinoza \& Hirano, 2003). Quanto ao nível de influencia dos atributos sobre a decisão de compra de um produto, os atributos são classificados de três maneiras (Espartel, 1999):

1) Atributos salientes: são os atributos que os consumidores conseguem perceber, porém que não inferem nenhum grau de importância na tomada de decisão de compra. Por exemplo, o design das garrafas de vinho pode ser percebido pelos consumidores, mas pode ser insignificante na hora de escolher um vinho.

2) Atributos importantes: são avaliados como importantes no momento da compra, mas não determinam a escolha do produto a ser comprado. Deste modo não agregam valor a compra, pois estão presentes em uma categoria similar de produtos (Tibola, 2004).

3) Atributos determinantes: são os atributos considerados importantes e capazes de influenciar a decisão de compra, pois são percebidos pelo consumidor como sendo a melhor opção para satisfazer seus desejos e necessidades (Espinoza \& Hirano, 2003). Por exemplo, na hora de escolher o vinho que irá acompanhar o jantar em um restaurante, a harmonização da bebida com o prato escolhido pode ser determinante para a escolha do produto.

\section{ATRIBUTOS DO VINHO}

Com relação ao consumo de vinhos, estudos em diferentes contextos investigaram os atributos que podem influenciar o comportamento de escolha do consumidor. Goodman et al. (2007) identificaram treze elementos que influenciaram o comportamento de consumo em países como Austrália, Áustria, Brasil, França, Itália, Nova Zelândia e Estados Unidos. Dos treze itens identificados, oito eram atributos do produto: variedade da uva; origem do vinho; marca; premiação; harmonização com alimentos; informações contidas no contra-rótulo; rótulo frontal atraente; teor alcoólico.

Existem no mundo milhares de variedades de uvas. Para Galvão (2004) as uvas podem ser classificadas em duas categorias: uvas não-viníferas, também chamadas uvas de mesa, e uvas viníferas, próprias para a elaboração de vinhos finos. As variedades de uvas presentes nas regiões tradicionais na produção de vinhos como França, Itália, Portugal e Espanha, foram selecionadas naturalmente ao longo do tempo e adaptadas às condições locais de solo, clima, e trato cultural. Com o passar do tempo essas espécies de videiras foram levadas para outros lugares, a exemplo de países do Novo Mundo. Em alguns casos a adaptação da espécie foi perfeita, às vezes até excedendo a qualidade das cepas originais, em outros, os frutos evoluíram criando características que fogem de sua 
tipicidade original (Pinto, 2009).

O país de origem é outro atributo que exerce influência na avaliação de um produto (Guilhoto, 2001; Giraldi \& Ikeda, 2007). As pessoas têm imagens estereotipadas de cada país, e usam essas imagens como fontes de informações para auxiliar na escolha do produto que vão adquirir. O país de origem é considerado um fator importante para os compradores de vinho, pois o produto tem ligações com sua origem. No entanto, uma mesma variedade de uva cultivada em diferentes regiões produz vinhos com diferentes sabores (Lattey et al., 2007).

A marca é outro critério de avaliação muito importante, pois reflete o valor significativo que cada produto tem para os seus consumidores (Ruão \& Farhangmer, 2000), além de ser a fonte principal de vantagem competitiva para uma organização (Louro, 2000). A imagem que o consumidor tem sobre determinada marca é construída através de um conjunto de valores funcionais, econômicos e emocionais ou psicológicos (Barbosa, 2009; Louro, 2000).

As medalhas e prêmios também são importantes atributos de influencia na preferência do consumidor, pois servem como indicador de qualidade do produto (Lockshin \& Hall, 2003). Quando um vinho é premiado significa afirmar que ele foi avaliado favoravelmente por especialistas em relação a outros vinhos. Isso sinaliza ao comprador que o vinho é de alta qualidade e é capaz de ser positivamente percebido por outras pessoas (Mueller, 2009).

A harmonização do vinho com os alimentos tem o objetivo de valorizar o sabor de ambos. Quando em harmonia, vinho e comida propiciam um prazer muito maior do que quando degustados separadamente (Queiroz, 2009). Entretanto, para um casamento perfeito, vinho e alimento precisam ser compatíveis. Para tanto, é necessário conhecer bem sobre vinhos e saber a preparação do prato com o qual o vinho será harmonizado (Pinto, 2009).

Pela grande variedade de vinhos ofertados, alguns consumidores se veem confrontados com muitas decisões. Para ajudálos nessa escolha, as informações encontradas no contra-rótulo os auxiliam a conhecer mais sobre determinado vinho (Lockshin \& Hall, 2003). Segundo Mcgarry \& Thompson (2010), os contra-rótulos que contém informações sobre os sabores e aromas do vinho influenciam mais do que outros sem esses dados.

O álcool é um importante componente do vinho, pois é responsável pela sua conservação e qualidade além de proporcionar uma sensação de queimação e calor ao ser degustado. Quanto maior o teor alcoólico do vinho maior será a percepção dessas sensações. A informação sobre a graduação alcoólica do vinho está sempre presente no rótulo, ela pode estar expressa em \% ou GL, podendo variar de 7 à 15\% (ou 7 á 15 oㄴ) do total (Pinto, 2009), exceto em vinhos fortificados como o Vinho do Porto, elaborado a partir da aguardente vínica que tem o teor alcoólico por volta de 20 a $22 \%$ GL (ou 20 à 22\%) (Miranda, 2001).

Outro atributo importante, porém não relatado na pesquisa de Goodman et al. (2007), é o preço. Dentre todos os produtos 
que os consumidores já adquiriram no decorrer de suas vidas, vários deles foram escolhidos pelo valor que custavam (Engel et al. 2000) tendo um forte grau de influência na decisão de compra de vinhos (c.f Magistris et al., 2011; Guerra, 2005; Johnson \& Bastian, 2007; Mcgarry \& Thompson, 2010).

\section{COMPORTAMENTO DO CONSUMIDOR DE VINHO}

Para enólogos, a qualidade do vinho está relacionada a determinados parâmetros traçados para a produção dessa bebida, tais como método de plantio da uva, quantidade de açúcar do fruto, entre outros. Essa percepção de qualidade difere da do consumidor, que considera suas preferências e gostos individuais ao classificar um vinho como sendo de qualidade. Ao analisar as preferências do consumidor, as equipes de marketing e de produção podem direcionar os tipos de vinhos para diferentes categorias de consumidores, ou reposicionar uma marca existente de vinho para melhorar as vendas. Pode-se também verificar se o consumidor prefere mais ou menos acidez, se está mais sensível a escolher um vinho pelo seu preço, ou pela marca, etc. (Lattey et al., 2007).

Na França, país com tradição em produção e consumo de vinhos, os três atributos mais citados como relevantes na escolha da bebida foram respectivamente: harmonização com a comida, país de origem do vinho e conhecimento prévio do produto. Na Itália, os três itens preferidos foram: degustação prévia do vinho, harmonização com a comida e país de origem, respectivamente (Goodman et al., 2007). Já na Espanha, o fator preço foi avaliado positivamente, embora os consumidores importaram-se mais com a harmonização entre vinho e a comida e o país de origem (Magistris et al., 2011). Em Portugal, os consumidores de vinho tenderam a escolher vinhos com preços inferiores (Guerra, 2005). Entretanto, os consumidores Húngaros valorizaram mais o sabor, a qualidade e o país de origem do vinho (Szabo \& Szeles, 2006). Os atributos mais valorizados pelos austríacos foram: variedade de uva, degustação prévia do vinho e país de origem (Goodman et al., 2007). Na Austrália, os atributos mais importantes na decisão de compra de um vinho foram: conhecimento prévio do vinho, recomendado por alguém e variedade da uva (Goodman et al., 2007). Uma análise mais detalhada por tipos de consumidores indicou que a região de origem e a reputação do vinicultor foram mais importantes para consumidores de alta especialização em vinho, enquanto que para os consumidores menos conhecedores da bebida, o estilo de vinho e o preço tiveram mais relevância para escolha do produto (Johnson \& Bastian, 2007).

Nos Estados Unidos, o conhecimento prévio do vinho (Goodman et al., 2007), a recomendação por alguém (Goodman et al., 2007; Lewis, 2010), a variedade de uva (Goodman et al., 2007; Lewis, 2010) e o país de origem do vinho (Lewis, 2010) são os fatores que mais influenciam na decisão de compra do consumidor de vinho. Enquanto no Brasil, a marca foi citada como atributo de maior importância na escolha de um vinho, seguida de 
conhecimento prévio do vinho e harmonização com a comida (Goodman et al., 2007).

Após revisão de pesquisas realizadas em outros países, a próxima seção apresenta a metodologia aplicada para identificar a relevância dos atributos do vinho na compra do produto para o consumidor da RMR, ao expor a abordagem de pesquisa, os instrumentos de coleta de dados, os procedimentos para definição da amostra, além da técnica empregada para análise dos dados, bem como as limitações da pesquisa.

\section{METODOLOGIA}

Ao observar estudos realizados em outros países, percebe-se que a abordagem quantitativa tem sido a mais utilizada para pesquisar as preferências dos consumidores em relação aos atributos de um produto. Dentro dessa abordagem, os métodos mais empregados foram o Ranking, o Método do Emparelhamento e a escala Likert. Entretanto, mais recentemente, a escala Best-Worst tem sido sugerida, pois permite estudar um grande conjunto de atributos de maneira mais rápida e fácil, evitando a fadiga dos entrevistados que pode gerar resultados inconsistentes e distorcidos (Groot, 2011).

Por meio do instrumento Best-Worst Scaling (ou BWS, inventada por Jordan Louviere em 1987, é também chamada de Maximum Difference Scaling, ou MaxDiff), os pesquisados são convidados a indicar entre as alternativas oferecidas o melhor, ou o mais importante, e o pior, ou o menos importante atributo do produto (Groot, 2011). Deste modo, consegue-se identificar a diferença máxima entre as preferências ou o grau de importância que o consumidor dá a determinado atributo (Erdem et al., 2010). Nesse método os entrevistados não podem gostar ou não gostar de todos os atributos. Sua intenção é identificar o peso de importância resultante que cada atributo tem em relação aos outros (Lockshin et al., 2009).

Os questionários da BWS são relativamente fáceis de serem entendidos pela maioria dos entrevistados, pois as pessoas julgam mais facilmente os itens quando esses são situados em extremos do que avaliá-los por meio de escalas que os ordenam em diferentes graus de importância ou preferência (Sawtooth Software, 2007). A propriedade importante do método é que a escala é livre e não há estilo de resposta, aspecto relevante para pesquisas transculturais (Sawtooth Software, 2007). Graças a estes benefícios, este método vem sendo utilizado em vários trabalhos sobre o comportamento do consumidor.

Para atingir os objetivos deste trabaIho foi elaborada uma escala de atributos do vinho com base na pesquisa de Goodman et al. (2007). Foram considerados os seguintes atributos: variedade da uva; origem do vinho; marca; premiação; harmonização com alimentos; informações contidas no contra-rótulo; rótulo frontal atraente e teor alcoólico. Os itens - conhecimento prévio do vinho; recomendação por alguém; visor promocional na loja; informações na prateleira e leitura sobre o vinho - foram retirados por não serem considerados atributos do produto. Optou-se também por inserir na pesquisa o item preço por este ter sido apontado em ou- 
tras estudos como tendo alto grau de influencia na decisão de compra (c.f Magistris et al., 2011; Guerra, 2005; Johnson \& Bastian, 2007; Mcgarry \& Thompson, 2010), totalizando assim nove atributos a serem mensurados.

\section{INSTRUMENTO DE COLETA DE DADOS}

O instrumento de coleta de dados utilizado nesse trabalho foi o questionário com perguntas fechadas de múltipla escolha, adaptado das pesquisas de Forbes (2008) e Groot (2011) e elaborado pela ferramenta Survey Monkey (software de pesquisa online).

O resultado final foi um questionário com 15 questões divididas em três partes. A primeira parte foi composta por quatro questões sociodemográficas como idade, renda, educação e gênero além de uma questão sobre a frequência do consumo de vinho. A segunda parte foi composta por uma escala de intervalo, Likert, com 5 pontos, que variam de discordo plenamente (1) a concordo plenamente (5). A terceira e última parte é constituída por questões elaboradas com base na escala BW propriamente dita, em que os consumidores escolheram apenas o item mais importante e o menos importante entre as combinações possíveis dos atributos.

Para a construção da BWS é necessário determinar o tamanho do conjunto de escolha e a quantidade de combinações possíveis a serem feitas entre os atributos. Segundo Groot (2011) o experimento deve ter pelo menos três alternativas ou opções de escolha em cada conjunto e não ultrapassar seis alternativas, pois a qualidade de resposta do consumidor diminui quando o número de alternativas aumenta. Já Chrzan e Patterson (2006) recomendam um maior número de conjuntos com menor número de itens por questão e Orme (2005) propõe que o número de alternativas por conjunto de escolha não deve ser maior que à metade do número total de atributos, pois, hipoteticamente, um número maior de itens por conjunto resulta em menor precisão para os itens de importância ou preferência média.

Levando-se em consideração as observações feitas por Orme (2005), o número de alternativas por combinação utilizado nesse trabalho foi quatro. Como a metade do número total de atributos pesquisado (9) é igual a um número decimal $(4,5)$ e o número de alternativas do conjunto deve ser menor ou igual à metade do total de atributos cada questão do questionário apresentou um conjunto com quatro alternativas, o que também atende as recomendações de Groot (2011), Chrzan e Patterson (2006).

Para definir a quantidade de conjuntos de escolha foi usada uma função do número total de atributos avaliados (Ncsets, que quer dizer em inglês: number of choice sets).

$$
\begin{gathered}
\text { Figura } 2 \text { - Fórmula Ncsets } \\
\text { Ncsets }=k R / \mathrm{n}
\end{gathered}
$$

Fonte: Groot (2011, p. 107).

Na Figura 2, "k" representa o número total de atributos a serem estudados (9), " $n$ " é o número de alternativas em cada conjunto de escolha (4) e " $R$ " o número de vezes que cada atributo é apresentado através do conjunto de escolha a cada indivíduo, logo Ncsets $=9 \mathrm{R} / 4$. Mas ainda é necessário de- 
terminar "R". Conforme Groot (2011) não foram encontrados trabalhos de caráter científico que abordem essa questão e para evitar a fadiga dos entrevistados, ele decidiu repetir cada atributo 4 vezes através de conjuntos de escolha $(R=4)$, resultando em 9 combinações com 4 atributos cada, onde todos os atributos apareceram igual e repetidamente 4 vezes.

Com base nestas orientações elaboraram-se todas as combinações possíveis, ou seja, distribuir os nove atributos em conjuntos de escolha de forma equilibrada. Como cada atributo deve ter mesma chance de ser escolhidos, todos devem aparecer o mesmo número de vezes no questionário (Sawtooth Software, 2007).

\section{AMOSTRAGEM}

Para essa pesquisa os consumidores de vinho do Recife representam o universo. Dada a impossibilidade de pesquisar toda a população devido as limitações de tempo e recursos, a solução foi selecionar um grupo menor desse universo, mas que fosse o mais representativo possível. Segundo Gil (2002) a amostra adequada com um coeficiente de confiança de $95,5 \%$ para uma população de mais de 100.000 pessoas e com margem de erro de mais ou menos $10 \%$ é igual a 100 pessoas.

Após a montagem, pré-teste e revisão do questionário, os links de acesso ao software Survey Monkey foram enviados por email, e disponibilizados na rede social, via mensagens online ou convite de participação em evento.

\section{MÉTODO DE ANÁLISE DOS DADOS}

Para analisar os dados coletados pela escala BW, inicialmente calculou-se o nível de importância de cada atributo, que é o número de vezes que os entrevistados indicam cada atributo como mais importante (Countbest) menos o número de vezes que esse mesmo atributo foi apontado como menos importante (Countworst). 0 Countbest e Countworst encontrados para cada um dos nove atributos desse estudo estão expostos na Tabela 1:

Tabela 1 - Countbest e Countworst

\begin{tabular}{|l|r|r|}
\hline & Countbest & Countworst \\
\hline Variedade da uva & 195 & 40 \\
\hline Origem do vinho & 164 & 22 \\
\hline Harmonização com alimento & 184 & 65 \\
\hline Informações contidas no contra-rótulo & 132 & 37 \\
Preço & 71 & 83 \\
\hline Marca & 50 & 129 \\
\hline Premiação & 47 & 171 \\
\hline Teor alcoólico & 37 & 176 \\
Rótulo frontal & 20 & 177 \\
\hline
\end{tabular}

Fonte: Análise de dados 
A partir de então foi calculado o escore padrão, que é o nível de importância dividido pelo número de vezes que cada atributo aparece na combinação multiplicado pelo número de questionários respondidos, conforme pode-se observar na equação a seguir:

Figura 3 - Fórmula do escore padrão

Standard Score $=\frac{\text { Countbest }- \text { Countworst }}{4 n}$

Fonte: Goodman (2005, p. 6)

Após aplicação da equação, os resultados são expostos em tabelas na seção a seguir.

\section{RESULTADOS}

A amostra estudada foi dominada por consumidores com idade entre 18 e 35 anos e a quantidade de respondentes dos gêneros feminino e masculino foi equilibrada (47\% mulheres e 53\% homens). A maioria dos consumidores pesquisados possui curso superior completo (60\%) e no que se refere à renda familiar, a maioria dos respondentes (44\%), pertence à classe C, ou seja, possui renda entre 4 a 10 salários mínimos (SM) segundo a classificação de classe social adotada pelo Instituto Brasileiro de Geografia e Estatística (IBGE). Enquanto que a classe D (com renda familiar de 2 a 4 SM) é representada por $19 \%$ dos participantes da pesquisa. Em relação à frequência do consumo de vinho do Recife, grande parte dos consumidores bebe vinho apenas uma vez ao mês ou muito raramente.

\section{RELEVÂNCIA DOS ATRIBUTOS DO VINHO PARA OS CONSUMIDORES DO RECIFE}

Por meio da escala Best-Worst identificou-se o grau de relevância dos atributos em relação a sua influência sobre a decisão de compra de um produto. Foi adotada a classificação de Espartel (1999) que divide os atributos em: a) importantes - aqueles avaliados como importantes no momento da escolha do produto, mas que não determinam a compra do mesmo - e b) determinantes aqueles atributos capazes de influenciar a decisão de compra do consumidor.

Após análise dos dados coletados por meio da escala BW, 5 (cinco) dos 9 (nove) atributos investigados tiveram nível de relevância negativo e por isso foram classificados apenas como importantes (Espartel, 1999: 35). São eles: preço, marca, premiação, teor alcoólico e rótulo frontal atraente conforme tabela 2. 
Tabela 2 - Atributos do Vinho Classificados como Importantes pela Escala BW

\begin{tabular}{lcl}
\hline Atributos & Nível de relevância & Escore padrão \\
\hline Preço & -12 & $-0,03$ \\
Marca & -79 & $-0,1975$ \\
Premiação & -124 & $-0,31$ \\
\hline Teor alcoólico & -139 & $-0,3475$ \\
Rótulo frontal atraente & -157 & $-0,3925$ \\
\hline
\end{tabular}

Fonte: Análise de dados

O preço está entre os atributos indicados negativamente pela maioria dos respondentes, embora tenha sido o mais importante dentre os 5 atributos listados na tabela 2, pois obteve um escore padrão próximo de zero. Ao analisar as respostas dos itens que medem a importância do atributo preço sobre a decisão de compra do consumidor percebe-se que este realmente não é um atributo muito significativo para a amostra pesquisada conforme se pode observar na Tabela 3 .

Tabela 3 - Hipóteses exploratórias do atributo preço

\begin{tabular}{l|c|c|c|c|c|c}
\hline Hipotese exploratória & $\mathbf{1}$ & $\mathbf{2}$ & $\mathbf{3}$ & $\mathbf{4}$ & $\mathbf{5}$ & $\begin{array}{c}\mathbf{n} \mathbf{\text { de }} \\
\text { respostas }\end{array}$ \\
\hline Vinhos são caros & $12 \%$ & $23 \%$ & $\mathbf{3 6} \%$ & $\mathbf{2 4 \%}$ & $5 \%$ & 100 \\
\hline $\begin{array}{l}\text { Preço baixo é importante na compra de um } \\
\text { vinho. }\end{array}$ & $17 \%$ & $25 \%$ & $24 \%$ & $\mathbf{2 8} \%$ & $6 \%$ & 100 \\
\hline Não gosto de vinhos com alto teor alcoólico. & $19 \%$ & $18 \%$ & $29 \%$ & $21 \%$ & $13 \%$ & 100 \\
\hline $\begin{array}{l}\text { Já comprei vinho só por que apresentava um } \\
\text { rótulo frontal atraente. }\end{array}$ & $\mathbf{3 3} \%$ & $30 \%$ & $8 \%$ & $23 \%$ & $6 \%$ & 100 \\
\hline Prefiro tomar vinhos que já foram premiados. & $17 \%$ & $20 \%$ & $\mathbf{3 4 \%}$ & $22 \%$ & $7 \%$ & 100 \\
\hline Conheço muitas marcas de vinho. & $4 \%$ & $24 \%$ & $28 \%$ & $\mathbf{3 0} \%$ & $14 \%$ & 100 \\
\hline
\end{tabular}

Fonte: Análise dos dados

Apenas $29 \%$ dos respondentes acham os vinhos caros, enquanto que $35 \%$ deles discordam ou discordam plenamente dessa opinião. A baixa influência do atributo preço para os consumidores desta amostra fica ainda mais explicita quando os resultados apontam que $42 \%$ dos entrevistados discordam ou discordam totalmente que o preço baixo seja um aspecto importante na compra dessa bebida. Esse resultado é diferente do observado na pesquisa feita por Guerra (2005) em Portugal, onde os consumidores preferem comprar vinhos com preços baixos.

A marca do vinho e a premiação tam- bém foram atributos indicados negativamente pela escala BW não tendo um peso significante para os consumidores de vinho no momento da compra da bebida. Esse resultado pode ser indicativo de ausência ou de deficiência no plano de marketing dos produtores de vinho. A pouca divulgação e promoção das marcas e dos prêmios recebidos pelos vinhos pode dificultar o estabelecimento do produto no mercado, assim como a construção de uma relação de confiança com o consumidor a ponto de influenciá-lo na sua decisão de compra.

Analisando os resultados da escala $\mathrm{Li}$ - 
kert pode-se compreender melhor a percepção do consumidor sobre estes dois atributos. Apenas $44 \%$ dos consumidores concordam plenamente ou apenas concordam que conhecem muitas marcas de vinhos. E embora os consumidores conheçam um grande número de marcas, este aspecto não foi capaz de fazer com que o atributo marca tivesse seu nível de relevância classificado positivamente pelos respondentes. A pouca relevância da premiação fica evidente na sentença "Prefiro tomar vinhos que já foram premiados", pois os resultados indicam que apenas $29 \%$ dos consumidores concordam ou concordam totalmente com essa declaração.

O teor alcoólico do vinho também foi classificado como um atributo importante. Em relação às considerações dos consumidores em termos da graduação alcoólica da bebida, os resultados encontrados pela escala Likert revela que $29 \%$ dos consumidores são indiferentes quanto a esse atributo, enquanto que $34 \%$ não gostam de vinhos com alto teor alcoólico.

Por fim, o rótulo frontal atraente surge como último atributo importante. A baixa relevância do rótulo frontal também pode ser observada nos resultados encontrados pela escala Likert visto que $66 \%$ dos consumidores discordam ou discordam totalmente da afirmativa "Já comprei vinho só por que apresentava um rótulo frontal atraente".

Quatro dos nove atributos do vinho foram avaliados positivamente pelos consumidores pesquisados, e dessa forma foram considerados como determinantes para a compra da bebida, são eles: variedade de uva, origem do vinho, harmonização com alimento e informações do contra-rótulo, conforme resultados apresentados na Tabela 4.

Tabela 4 - Atributos do Vinho Classificados como Determinantes pela Escala BW

\begin{tabular}{lcc}
\hline Atributos & Nível de relevância & Escore padrão \\
\cline { 2 - 3 } Variedade da uva & 155 & 0,3875 \\
Origem do vinho & 142 & 0,355 \\
Harmonização com alimento & 119 & 0,2975 \\
Informações contidas no contra-rótulo & 95 & 0,2375 \\
\hline
\end{tabular}

Fonte: Analise de dados

Ao ponderar os atributos determinantes para os consumidores do Recife, a variedade de uva foi o atributo que mais influenciou na escolha do vinho. Interessante obser- var que os consumidores preferem as uvas tintas às brancas conforme as respostas da tabela 5 . 
Tabela 5 - Hipóteses exploratórias dos atributos determinantes

\begin{tabular}{|c|c|c|c|c|c|c|}
\hline Hipotese exploratória & 1 & 2 & 3 & 4 & 5 & $\begin{array}{c}\text { no de } \\
\text { respostas }\end{array}$ \\
\hline $\begin{array}{l}\text { Vinhos elaborados de uvas tintas são mais } \\
\text { saborosos. }\end{array}$ & $9 \%$ & $19 \%$ & $24 \%$ & $23 \%$ & $25 \%$ & 100 \\
\hline Eu gosto de beber vinho com a comida. & $3 \%$ & $8 \%$ & $10 \%$ & $42 \%$ & $37 \%$ & 100 \\
\hline $\begin{array}{l}\text { Eu acho o vinho mais gostoso quando } \\
\text { harmonizando com a comida. }\end{array}$ & $4 \%$ & $7 \%$ & $23 \%$ & $29 \%$ & $37 \%$ & 100 \\
\hline $\begin{array}{l}\text { Eu leio as informações do contra-rótulo para } \\
\text { comprar um vinho. }\end{array}$ & $7 \%$ & $10 \%$ & $20 \%$ & $34 \%$ & $29 \%$ & 100 \\
\hline $\begin{array}{l}\text { As informações do contra-rotulo me ajudam a } \\
\text { conhecer o vinho melhor. }\end{array}$ & $3 \%$ & $5 \%$ & $18 \%$ & $46 \%$ & $28 \%$ & 100 \\
\hline $\begin{array}{l}\text { Os vinhos internacionais são mais gostosos } \\
\text { que os vinhos brasileiros. }\end{array}$ & $16 \%$ & $17 \%$ & $35 \%$ & $24 \%$ & $8 \%$ & 100 \\
\hline $\begin{array}{l}\text { Os vinhos brasileiros são tão bons quanto os } \\
\text { importados. }\end{array}$ & $3 \%$ & $26 \%$ & $30 \%$ & $23 \%$ & $18 \%$ & 100 \\
\hline $\begin{array}{l}\text { Os vinhos tintos são mais fáceis de harmonizar } \\
\text { que os brancos. }\end{array}$ & $10 \%$ & $25 \%$ & $29 \%$ & $23 \%$ & $13 \%$ & 100 \\
\hline
\end{tabular}

Fonte: Analise dos dados

A origem do vinho é a segunda característica determinante para o consumidor no momento da compra. Entretanto, o consumidor não tem uma opinião formada e muito clara sobre a qualidade dos vinhos nacionais versus internacionais como se pode observar nos resultados obtidos pela escala Likert. $\mathrm{Na}$ sentença "Os vinhos internacionais são mais gostosos que os vinhos brasileiros", $32 \%$ dos consumidores pesquisados concordam ou concordam plenamente, enquanto que 33\% discordam ou discordam totalmente. Por outro lado, $41 \%$ dos respondentes concordam ou concordam plenamente que os vinhos brasileiros são tão bons quanto os importados.

O terceiro atributo determinante foi à harmonização com o alimento, confirmado pelo resultado das hipóteses exploratórias da escala Likert, visto que $79 \%$ dos respondentes gostam de beber vinho com a comida e $66 \%$ acham o vinho mais gostoso quando harmonizado com a comida. Embora esses resultados indiquem que o consumidor valoriza a harmonização, não foram encontrados indícios de que ele possui conhecimento suficiente para fazer essa combinação corretamente. Essa suspeita se intensifica ao observar a escala Likert, pois os consumidores têm dúvida sobre alguns aspectos importantes na harmonização. Apenas 35\% dos consumidores demonstram ter conhecimentos de que os vinhos tintos são mais difíceis de harmonizar, ao discordarem ou discordarem totalmente da frase "Os vinhos tintos são mais fáceis de harmonizar que os brancos".

As informações do contra rótulo foi o quarto atributo determinante para o consumidor no momento da compra. De acordo com os resultados encontrados pela escala Likert, 63\% dos respondentes leêm as informações contidas no contra rótulo antes de comprar o vinho e $74 \%$ consideram que essas informações ajudam a conhecer o vinho meIhor. Este achado corrobora os argumentos de Lockshin \& Hall (2003) de que os consumi- 
dores usam as informações do contra rótulo para escolher um vinho. Além disso, a relevância do contra rótulo para os consumidores de vinho comprova as afirmações de Mello (2002) e Sloan (2005) sobre a necessidade da busca de conhecimento do consumidor para apreciar melhor a bebida.

\section{CONCLUSÕES}

O destaque de aspectos conclusivos emergentes na análise dos dados nos faz retornar ao objetivo geral deste estudo: identificar e analisar a relevância dos atributos do vinho no processo decisório de compra desse produto para os consumidores do Recife.

Variedade de uva, origem do vinho, harmonização com o alimento e informações contidas no contra rótulo, foram respectivamente, os atributos determinantes para o consumidor de vinho do Recife em sua decisão de compra, ou seja, tais atributos tiveram maior capacidade de influenciar a escolha do consumidor. Comparando esses resultados com a pesquisa feita no Brasil por Goodman et al. (2007) os atributos harmonização do vinho com comida, a origem do vinho e a variedade da uva também aparecem entre os atributos mais relevantes. Entretanto, no estudo realizado por aqueles pesquisadores, a marca surge como atributo determinante no momento da compra - resultado divergente do levantamento realizado com os consumidores do Recife. No estudo desenvolvido na capital pernambucana, as informações do contra rótulo apareceram entre os atributos determinantes, enquanto que nos achados de Goodman et al. (2007) este aspecto foi indicado negativamente.
Dois atributos são destacados como determinantes tanto nos países pesquisados por Goodman et al. (2007) (Austrália, Áustria, Itália, França, Nova Zelândia, Estados Unidos da America, inclusive o Brasil) como no Recife e também em pesquisas de outros autores como Magistris et al. (2011), Szabo e Szeles (2006), Johnson e Bastian (2007), Lewis (2010) e McGarry e Thompson (2010). São eles: a variedade da uva e a origem do vinho. Estes dois atributos possuem uma interligação, pois plantando-se uma mesma casta de uva em lugares diferentes obtêm-se vinhos de sabores distintos. Segundo Forbes (2008: ii) há uma relação entre a origem do vinho e a percepção de qualidade do produto do consumidor. A variedade da uva é um atributo muito importante na elaboração da bebida, pois é responsável pelo aroma e sabor do vinho (Pinto, 2009: 44).

A variedade da uva e a origem do vinho, atributos peculiares do vinho, são atributos determinantes em vários países, que devem ser considerados pelos produtores para elaboração de seus vinhos. Mas é necessário pesquisar mais profundamente qual o país e a variedade de uva mais consumida no Recife, pois não foi observada uma opinião clara sobre a qualidade dos vinhos nacionais e internacionais apesar de o país de origem do vinho ter sido o segundo atributo mais relevante. Essas informações devem ser consideradas pelos produtores de vinho, assim eles conhecerão a preferência do consumidor em termos de origem do vinho e variedade de uva, para ajudá-los na elaboração do seu plano estratégico e de marketing.

Embora o preço do produto seja frequentemente usado pelos consumidores 
para auxiliar a sua decisão de compra (Engel et al. 2000), nessa pesquisa assim como nas pesquisas de Goodman et al. (2007) o preço foi indicado negativamente tendo pouca relevância para o consumidor de vinho. Diferente de algumas pesquisas onde o preço foi um atributo fundamental na compra de um vinho (Guerra, 2005; Mcgarry \& Thompson, 2010) ou foi indicado positivamente (Magistris et al., 2011). Os consumidores provavelmente utilizam o preço como delimitador importante de suas opções de escolha, eles consideram uma faixa de preço aceitável para comprar um vinho, porém esse atributo não é determinante no momento da compra dessa bebida (Forbes, 2008).

Johnson e Bastian (2007) fizeram uma observação importante em relação à influência do preço. Em suas pesquisas o preço baixo foi fator determinante apenas para os consumidores que tinham menos conhecimento sobre o vinho. Apesar de a relação entre preço e nível de conhecimento sobre o vinho não ter sido examinada neste estudo, podese perceber que o consumidor recifense está se aperfeiçoando na arte do vinho, preferindo vinhos finos aos de mesa, buscando mais conhecimento sobre a bebida, lendo as informações do contra rótulo e apreciando a harmonização com a comida.

Os achados desse estudo podem ser relevantes para os produtores e comerciantes de vinho desenvolverem estratégias capazes de influenciar seus consumidores, principalmente por que o mercado mundial do vinho está cada vez mais competitivo (Forbes, 2008).

Concluiu-se que muito dos atributos do produto não são determinantes para os consumidores durante suas decisões de compra de vinho. Atributos como a aparência do rótulo e premiação tiveram pouca influência sobre a decisão do consumidor. Esse resultado pode indicar que tais atributos atualmente recebem pouco esforço da equipe de marketing.

A continuidade dessa pesquisa pode ajudar a indústria na produção de vinhos que satisfaçam o perfil de seus consumidores, minimizando o risco de perder os clientes existentes, além de identificar quais marcas de vinhos são seus concorrentes. Assim, sugerese: 1) Elaborar pesquisas que levem em consideração a segmentos específicos de mercado, para que as empresas conheçam meIhor seus consumidores e seu público alvo e consigam planejar seus produtos e propagandas eficientemente; 2) Pesquisar as preferências do consumidor de acordo com as situações de consumo, que influenciam seu comportamento; 3) Aprofundar o estudo sobre algum atributo específico. Por exemplo, o país de origem foi um atributo relevante para os consumidores, mas seria interessante identificar qual ou quais países o consumidor prefere no momento de comprar um vinho. Pode-se pesquisar também quais as informações que o consumidor gostaria de encontrar no contra-rótulo, um dos atributos mais relevantes nessa pesquisa, pois segundo Mcgarry e Thompson (2010) os rótulos que continham informações sobre os sabores e aromas do vinho tiveram impacto mais positivo sobre a decisão de compra dos consumidores.

\section{REFERÊNCIAS}

Barbosa, C. S. (2009) “Notoriedade e valor da marca 
dos vinhos verdes." Dissertação de Mestrado em Gestão Comercial, Faculdade de Economia, Universidade do Porto, Porto.

Blackwell, R. D.; Miniard, P.W. \& Engel, J. F. (2009) "Comportamento do consumidor". Cengage Learning, São Paulo

Cardeira, R.F.F. (2009) "Factores Críticos de Sucesso no Mercado do Vinho em Portugal e a Sustentabilidade do Sector Vitivinícola". Dissertação de Mestrado em Engenharia e Gestão e Industrial, Universidade Técnica de Lisboa, Lisboa

Chrzan, K. \& Patterson, M. (2006) "Testing for the Optimal Number of Attributes in MaxDiff Questions". Sawtooth Software. Disponível em: http://www.sawtoothsoftware.com/download/techpap/mdoptimalatts.pdf. Acessado em 20 de maio de 2012

Churchill, G.A. \& Peter, P. (2005) "Marketing criando valor para os clientes". Saraiva, São Paulo

Engel, J. F.; Blackwell, R.D.\& Miniard, P. W. (2000) "Comportamento do Consumidor". Livros Técnicos e Científicos Editora S.A., Rio de Janeiro

Erdem, S.; Rigby, D. \& Wossink, A. (2010) “Who is most responsible for ensuring the meat we eat is safe?" Ageconsearch, Edinburgh, http://ageconsearch.umn.edu/handle/91813. Acessado em 10 de abril de 2010

Espartel, L. B. (1999) "Atributos de produto e motivações de compra no mercado jornalístico do Rio Grande do Sul". Dissertação de Mestrado em Administração, Universidade Federal do Rio Grande do Sul, Porto Alegre

Espinoza, F. da S.; Hirano, A.S. (2003) "As dimensões de avaliação dos atributos importantes na compra de condicionadores de ar: um estudo aplicado" RAC, 7(4): 97-117

Fernandes Neto, A.F. (2006) "A Importância da Globalização para as Empresas Brasileiras" THESIS, São Paulo, 5(3): 1-20, Disponível em: http://www.cantareira.br/thesis2/n5a3/alvaro.pdf. Acessado em 2 de março de 2012

Forbes, S. L. (2008) "The influence of individual characteristics, product attributes and usage situations on consumer behaviour: An exploratory study of the New
Zealand, Australian, UK and US wine markets" Dissertação de Doutorado em Filosofia em Marketing, Lincoln University

Galvão, S. (2004) "A cozinha e seus vinhos: Receitas rápidas com muita classe". Editora Senac, São Paulo

Gil, A.C. (2002) "Como elaborar projetos de pesquisa" Atlas, São Paulo

Giraldi, J. de M.E. \& IKEDA, A.A. (2007) "A Influência dos Valores Pessoais dos Consumidores no "Efeito país de Origem". Anais do XXXI ENANPAD. pp 1-16.

Goodman, S.; Lockshin, L. \& Cohen, E. (2005) “BestWorst scaling: A simple method to determine drinks and wine style preferences". Wine Preferences. Disponível em: http://www.winepreferences.com/resources/page28/files/page28_1.pdf. Acessado em 20 de junho de 2012

Goodman, S.; Lockshin, L. \& Cohen, E. (2007) "Influencers of consumer choice in a retail setting more international comparisons" Wine Industry Journal, 22(6): 42-48

Groot, E. (2011) "Choice experiments with best-worst alternatives to understand consumer behaviour: application to peaches with Protected Designation of Origin (PDO) Calanda". Dissertação de Doutorado em Economia, Facultad de Economía y Empresa, Universidad de Zaragoza, Zaragoza .

Guerra, N. J.M. (2005) “Análise do processo de decisão de compra do consumidor de vinho. - a avaliação de factores no contexto português". Dissertação de Mestrado em Gestão de Empresas, Universidade de Évora, Évora

Guilhoto, L. de F.M. (2001) "A influência do país de origem na percepção do consumidor sobre a qualidade dos produtos" Caderno de Pesquisas em Administração, 8 (4)

Hawkins, Del I.; Mothersbaugh, D.L. \& Roger, J. B. (2007) "Comportamento do consumidor: construindo a estratégia de marketing" Elsevier, Rio de Janeiro

IBRAVIN (2012a) "Importações brasileiras de vinhos e espumantes". Disponível em: http://www.ibravin.org.br/admin/UPLarquivos/160520121038402.pdf. Acessado em 8 de março de 2012 
IBRAVIN (2012b) "Vendas de vinhos crescem, mas estoques seguem altos". Disponível em: http://www.ibravin.org.br/int_noti-

cias. php id $=855 \&$ tipo $=N$. Acessado em 08 de março de 2012

Johnson, T. \& Bastian, S. (2007) "A preliminary study of the relationship between Australian wine consumers' wine Expertise and their wine purchasing and consumption behavior". Australian Journal of Grape and Wine Research, 13(3): 186-197

Kotler, Philip. (1998) “Administração de Marketing: análise, planejamento, implementação e controle"Atlas, São Paulo

Las Casas, A.L. (2008) "Administração de marketing: conceitos, planejamento e aplicações à realidade brasileira" Atlas, São Paulo

Lattey, K.A.; Bramley, B.R.; Francis, I. L.; Herderich, M.J. \& Pretorius, S. (2007) “Wine quality and consumer preferences: understanding consumer needs". Wine Industry Journal, 22(1):

31-39

Lewis, R.L. (2010) “Exploring consumer preferences in the United States wine market: Market segmentation applying best-worst scaling. Generation e Emphasis" Dissertação de Mestrado em Marketing, Department of Marketing and Statistics, Aarhus School of Business, Aarhus.

Lockshin, L.; Cohen, E. \& Goodman, S. (2009) "Overcoming Measurement Errors: Segmenting Wine Consumers across 11 Countries". Wine Industry Journal, 24 (1): $42-47$

Lockshin, L. \& Hall, J. (2003) “Consumer Purchasing Behaviour for Wine: What We Know and Where We are Going". Academy of Wine Business. Colloquium in Wine Marketing 2003. http://academyofwinebusiness.com/wp-content/uploads/2010/05/File-030.pdf. Acessado em 10 de abril de 2012

Louro, M.J.S. (2000) "Modelos de Avaliação de Marca". Rae - Revista de Administração de Empresas, 40(2): 26-37

Lunardo, R. \& Guerinet, R. (2007) "The influence of label on wine consumption: its effects on young consumers' perception of authenticity and purchasing behavior". Anais do CONGRESS EAAE Seminar. França, University of Reims, pp. 68-84.
Magistris, T.de.; Groot, E.; Gracia, A. \& Albisu, L. M. (2011) "Consumers preferences for wine in Spain: best-worst scaling methodology". Anais do CONGRESS $E A A$. Espanha, Unidad de Economía Agroalimentaria y de los Recursos Naturales

Malheiros, P. de G. (2006) "Saber beber, saber viver: estudo antropológico sobre as representações e práticas em torno do consumo de vinho entre degustadores, na cidade de Porto Alegre". Dissertação (Mestrado em Antropologia) 105f. Porto Alegre. Universidade Federal do Rio Grande do Sul

Marti, J.(2012) "A best-worst scaling survey of adolescents' level of concern for health and no health consequences of smoking". ECN, Seminá-

rios Matuszewski. Disponível em: http://www.ecn.ulaval.ca/seminaire/arti-

cle//a_best_worst_scaling_survey_of_adolescents_level_of_con-

cern_for_health_and_non_health_consequences/.

Acessado em 10 de abril de 2012

Mcgarry, M. W. \& Thompson, M. (2010) "The Importance of the information on the back label of a wine bottle on the purchase decision" Anais da AARES pre-conference workshops. Australia, Adelaide

MELLO, L.M.R de. (2002) "Tendência de Consumo e Perspectivas do Mercado de Vinhos no Brasil". Embrapa Uva e Vinho. Rio Grande do Sul. http://www.cnpuv.embrapa.br/publica/artigos/tendencia.pdf. Acessado em 05 de março de 2012

Miranda, F. (2001) "Arte e vinho." Axel Books do Brasil Editora, Rio de Janeiro

Mueller, Simone. et al. (2009) "How does shelf information influence consumers' wine choice?" The Australian Wine Research Institute. Disponível em: http://winepreferences.com/resources/page59/files/page59_1.pdf. Acessado em 15 de abril de 2012

ORME, Bryan. (2005) "Accuracy of HB Estimation in MaxDiff Experiments" Sawtooth Software. Disponível em: http://www.sawtoothsoftware.com/download/techpap/maxdacc.pdf. Acessado em 20 de maio de 2012

Pinto, D. (2009) “Manual didático do vinho: iniciação a enologia" Editora Anhembi Morumbi, São Paulo

Queiroz, S. J. de. (2009) "Mitos e controvérsias no cosmo da harmonização de vinhos" Trabalho de Con- 
clusão de Curso - Universidade do Vale do Itajaí

Roese, M. (2008) "O mondovino de cabeça para baixo: as transformações no mercado internacional do vinho e o novo empresariado vinícola". Revista de Sociologia e Política, 16(31): 71-83

Ruão, T. \& Farhangmer, M. (2000) “A imagem de marca: análise das funções de representação e apelo no marketing das marcas. Um estudo de caso" Disponível em: http://repositorium.sdum.uminho.pt/bitstream/1822/1985/3/truao_Farhangmer_CMark_2000.pdf. Acessado em 10 de abril de 2012

Sawtooth Software. (2007) "The MaxDiff/Web System Technical Paper" Disponível em: http://www.sawtoothsoftware.com/download/techpap/maxdifftech.pdf. Acessado em 20 de maio de 2012

Shef, J.N.; Mittal, B. \& Newman, B. I. (2001) "Comportamento do Cliente: Indo além do comportamento do consumidor". Atlas, São Paulo

Shiffman, L.G \& Kanuk, L. L. (2009) “Comportamento do consumidor". LTC, Rio de Janeiro

Sloan, D. (Org.).(2005) "Gastronomia, restaurantes e comportamento do consumidor". Manole, São Paulo

Stefano, N.; Capoval, A. \& Godoy, L. P. (2008) “Explorando conceitos e modelos sobre o processo de decisão de compra do consumidor em função da mudança de hábito alimentar". Anais do IX Congresso Nacional de Excelência em Gestão. Rio de Janeiro, pp.1-20

Szabo, Z. \& Szeles, Z. (2006) "Analysing Wine Buying Behaviour in Hungarian Hypermarkets" Anais da International Association of Agricultural Economists Conference. Gold Coast, Australia. Disponível em: http://ageconsearch.umn.edu/handle/25235. Acessado em 20 de abril de 2012.

Tibola, F.; Vieira, V. \& Sanzovo, J. (2004) “Atributos importantes na compra de notebooks: um estudo exploratório". Anais do VII SEMEAD. São Paulo

Urdan, F.T. \& Nagão, L. (2004) “Atributos intrínsecos e extrínsecos nas avaliações dos consumidores e efeitos na qualidade, valor e satisfação". Anais do XII SEMEAD. São Paulo
Wine Institute (2013a). World wine consumption by volume. Consultado el 06 de marzo, 2015, de http://www.wineinstitute.org/fi-

les/2010_World_Wine_Consumption_By_Volume_Ran̄k.pdf.

Wine Institute (2013b). World wine production by country. Consultado el 06 de marzo, 2015, de http://www.wineinstitute.org/fi-

les/2010_World_Wine_Production_by_Country.pdf.

Wines of Brasil. (2011) "Exportação de vinho brasileiro engarrafado cresce 33,6\% em 2011". Disponível em: http://www. winesofbrasil.com/Noticia.aspx?id=50.

Acessado 08 de março de 2012

Wines of Brasil (2012). Um grande país só poderia elaborar grandes vinhos. Consultado el 05 de septiembre, 2012, de em: http://www.winesofbrasil.com/Conteudo. aspx?id=4.

Zortea, C.G.C. (2010) "Luxo e vinho: Uma relação refletida pela revista Veja". Monografia de Graduação em Comunicação Social. Faculdade de Biblioteconomia e Comunicação, UFRGS

Dados dos Autores

\section{Nayara Diniz}

Graduada em Hotelaria, Administração, Negócios e Marketing pela Universidade Federal de Pernambuco. Email: nay tcd@hotmail.com

\section{Simone Almeida}

Mestre e Doutora em Administração. Professora do Departamento de Hotelaria e Turismo da Universidade Federal de Pernambuco. Email: simoneufpe.almeida@gmail.com

\section{Viviane Salazar}

Mestre e Doutora em Administração pela Universidade Federal de Pernambuco. Professora do Departamento de Hotelaria e Turismo da Universidade Federal de Pernambuco. Email: viviane salazar@yahoo.com.br

\section{Anderson Gomes de Souza}

Doutor e PHD em Administração. Professor do Departamento de Hospitalide e Turismo na Universidade Federal de Pernambuco (UFPE). Email: son ander@hotmail.com 